\title{
Numerical Simulation of Air-Water Slug Flow in the Aeration Diffuser Pipe of a Membrane Bioreactor
}

\author{
Guiqin Liu, Hui An, Peng Cheng Wang, Joo Guan Hang, Simon C. M. Yu
}

\begin{abstract}
In the membrane filtration system of the membrane bioreactor, air is used to scour the membrane. Liquid slug is not desirable as it will cause non-uniform aeration flow through all orifices. Computational Fluid Dynamics (CFD) simulations to predict the onset of air-water slug flow in the aeration diffuser pipe have been performed in this paper using the Volume of Fluid (VOF) method. The effects of various geometrical parameters on aeration diffuser pipe are investigated subsequently to predict the critical gas velocities at the onset of slug flow. Through dimensional analysis, the non-dimensional critical gas flux is found to be strongly correlated to the geometrical parameters of aeration diffuser pipe (area ratio of nozzle outlet and aeration pipe inlet). Through the parametric studies conducted in this work, an empirical model was developed and established for future design of aeration systems that can potentially prevent the intermittency of air bubbles caused by slugging.
\end{abstract}

Keywords - Two-Phase Flow, Slug Flow, CFD, Aeration Pipe, Membrane Bioreactor (MBR).

\section{INTRODUCTION}

$\mathrm{T}$ HE membrane bioreactor (MBR) includes a conventional biological treatment system and membrane filtration system. In the membrane filtration system, the aeration cleaning method uses air diffuser to scour the membrane. Energy cost is significant in the filtration system due to compressed air cost. In aeration diffuser system, air is pumped into the diffuser pipes from both sides, where the aeration holes are positioned along the diffuser pipes. Air flows in the pipes and exit through aeration holes in the form of bubbles. Due to buoyancy forces, air bubbles will rise towards the membrane system, which is placed above the diffuser pipes. Air bubbles would scour the surface of membrane by creating shear forces. Therefore, the process of generating air bubbles from air diffuser system is important on the performance of overall MBR system. Generating uniform aeration flow in a diffuser pipe is therefore critical as it helps to achieve on equal distribution of bubbles and subsequent air scouring process in the MBR system [1].

Liquid slugging is one of the main factors that cause the nonuniform aeration flow and uneven liquid height along the horizontal pipe. Previous studies have shown that whenever an interface exists between the gas flow over a liquid surface in a horizontal pipe, the interface produces wave-like behavior that leads to the formation of liquid slug [1]-[3]. The Kelvin

Guiqin Liu is with the Singapore Institute of Technology, Singapore (phone: 65-65921063; e-mail: guiqin.liu @ singaporetech.edu.sg).

Hui An is with the Singapore Institute of Technology, Singapore (phone: 65 65922074; e-mail: hui.an@ singaporetech.edu.sg).

Peng Cheng Wang is with the Singapore Institute of Technology, Singapore (phone: 65-65928510; e-mail: victor.wang@ singaporetech.edu.sg).
Helmholtz instability is found to be the basic mechanism of slug formation when the suction effect due to the pressure variation over a wave becomes sufficiently large to overcome the stabilizing effect of gravity. With the assumptions of ideal flow, negligible viscosity effect, constant pressure on the free surface and small amplitude over long wave, the criterion for the classical Kelvin-Helmholtz instability is given theoretically by:

$$
U_{g}-U_{l} \geq \sqrt{\frac{\left(\rho_{l}-\rho_{g}\right) g H_{g}}{\rho_{g}}}
$$

In the diffuser pipe, both the gas velocity and pipe geometry are significant to the flow. Firstly, the gas velocity affects the formation of waves in the pipe. The waves will be formed when the gas velocity increases, and eventually develop into wave coalescence and hence slugging [1]. Secondly, the gas flow rate affects the water level in the pipe which influences the amount of gas flowing out from the aeration holes [2]. The higher gas flow rate produces uniform water level resulting in constant pressure in the diffuser pipe [4]. Finally, the geometrical parameters of the aeration hole and pipe are also critical to the flow. Smaller holes increase the pressure drop at the aeration hole, causing lower liquid height in the pipe. The position of aeration holes position is also shown to affect the gas-liquid interface and the liquid height [1].

In conclusion, lower flow rate can reduce the operation cost, but it may cause slugging in diffuser pipes and non-uniform flow rate at aeration holes.

In the present work, CFD simulation is adopted to study the effects of various geometrical parameters on the onset of slug flow in aeration pipe. From MBR industry perspective, the objective is to ensure the aeration pipe can operate steadily in stratified flow conditions, prevent slug from forming. The stable operation of diffuser pipe would enhance the air scouring on the filters and hence prolong their operating lifespan. This study aims to identify the important parameters of diffuser pipe which could potentially prevent the occurrence of slug flow.

\section{VALIDATION}

The experiment conducted by Sato et al. [1] was chosen for the validation study. The aeration diffuser pipe was submerged in the liquid depth of $290 \mathrm{~mm}$ initially. The gas was injected

Joo Guan Hang is with the Singapore Institute of Technology, Singapore (phone: 65-65922144; e-mail: jooguan.hang@ singaporetech.edu.sg).

Simon C.M. Yu was with the Nanyang Technological University, Singapore and Singapore Institute of Technology, Singapore. Now he is with The Hong Kong Polytechnic University, Hong Kong (e-mail: simon.c.yu@ polyu.edu.hk) 
into the diffuser pipe with a flowmeter and pressure gauge being placed to monitor the gas flow rate. The gas would drive the liquid in the diffuser pipe and depart from the nozzles. The diameter and length of the diffuser pipe were $20 \mathrm{~mm}$ and 260 $\mathrm{mm}$ respectively, while the diameter of the five nozzles was 5 $\mathrm{mm}$. These nozzles were distributed evenly on top of the diffuser pipe, and $50 \mathrm{~mm}$ away from each other.

The experiments were performed with an inlet air flow rate of $7.0 \times 10-4 \mathrm{~m}^{3} / \mathrm{s}$ at $25^{\circ} \mathrm{C}$. The flow rate from each aeration hole was measured by dividing the collected air bubble volume by the capturing time. The measurement was repeated 15 times for each hole to obtain an averaged value [1]. The measured flow rate will be compared with the CFD results in the validation section.

The numerical results of air flow rate passing through each nozzle were compared with the experimental results, as shown in Fig. 1. The averaged value along $1 \mathrm{~s}$ after quasi-steady was used as the flow rate at the corresponding nozzle, while the standard deviation is treated as the uncertainty. As shown in Fig. 1, the maximum discrepancy between numerical and experimental flow rates for all nozzles was observed to be about $7.5 \%$ at the $5^{\text {th }}$ nozzle, within acceptable range. It can be concluded that the numerical results were validated.

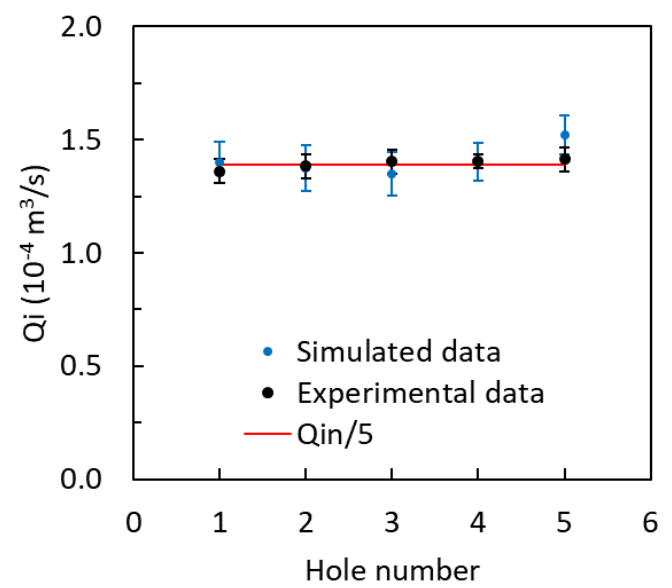

Fig. 1 Comparison of air flow rate on each nozzle between simulated results and Sato et al. (2017)'s experimental results [1]

\section{GRID INDEPENDENCE STUDY}

In the grid independence study, the flow rate at the first nozzle (counting from the inlet) is deemed a critical point for data to be collected. Concurrently, the distribution of air volume fraction on symmetry plane along the main pipe was also reviewed to guarantee a correct flow phenomenon.

In the grid independent study, the mesh size near nozzles was $1 \mathrm{~mm}$, and the other was $2 \mathrm{~mm}$ for the axial medium mesh case. For the axial fine mesh, the mesh size of the axial fine case was half of the axial medium, while the mesh size was doubled for the axial coarse mesh. Illustration of the meshes in the axial direction was shown in Fig. 2. No significant differences were observed at the air-water interface for the three different mesh refinement. However, the fluctuation of the air flow rate on the coarse mesh is $59 \%$ of the averaged flow rate, much larger than the medium mesh. The high fluctuation could cause instability. Hence, the medium mesh will be chosen in this paper.

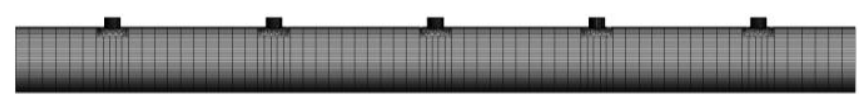

(a) coarse mesh

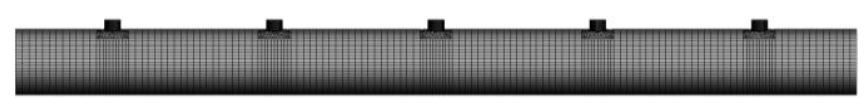

(b) medium mesh

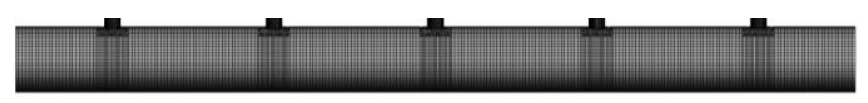

(c) fine mesh

Fig. 2 Axial meshes on the pipe wall

\section{NUMERICAL METHODS}

The simulations were carried out using ANSYS Fluent 18.2. The Volume of Fluid (VOF) method was employed to solve the volume fraction of air and water, and the air-water interface is computed based on interface interpolation treatment to the cells that lie near between different phases. The continuity equation, as shown in (2), for incompressible flow is solved for each phase as conservation of volume fraction. The implicit scheme was chosen to discretize volume fraction equation as in (3). The momentum conservation of the mixture was solved throughout the domain (4). The concept of mixture for two phases was introduced and the density of mixture was calculated based on (5). This mixture concept is also applicable to viscosity. The approach of Unsteady Reynolds Averaged Navier-Stokes (URANS) model along with the Boussinesq hypothesis was employed to the continuity and momentum equations. In order to solve the Reynolds-averaged quantities, the Realizable k- $\varepsilon$ turbulence model was chosen as a closure turbulence model for the Reynolds Stress term.

The continuous equation is:

$$
\frac{\partial\left(\alpha_{q}\right)}{\partial t}+\nabla\left(\alpha_{q} \overrightarrow{V_{q}}\right)=0
$$

where $q$ is the phase. For this paper, q could be gas or water. $\alpha$ is volume fraction, $\mathrm{V}$ is the velocity vector.

Implicit discretization of volume fraction is determined by:

$$
\frac{\alpha_{q}^{n+1}-a_{q}^{n}}{\Delta t} V+\sum_{f}\left(U_{f}^{n+1} \alpha_{q, f}^{n+1}\right)=0
$$

where $\mathrm{V}$ is the volume of the cell.

The momentum equation is:

$$
\frac{\partial \vec{V}}{t}+(\vec{V} \cdot \nabla) \vec{V}=-\frac{\Delta P}{\rho}+\nabla\left[\frac{\mu_{e f f}}{\rho}\left(\nabla \vec{V}+\vec{V}^{T}\right)\right]+\frac{\overrightarrow{F_{S}}}{\rho}+g
$$

where the density of the mixture is defined as

$$
\rho=\alpha_{g} \rho_{g}+\alpha_{l} \rho_{l}
$$


The simulation is treated as transient, where the diffuser pipe is to be filled with water initially. Air is injected from the inlet boundary into the simulation domain as the velocity inlet, and the nozzle outlets are defined as pressure outlet. The time step is set as $1 \mathrm{~ms}$, which has been shown sufficient to reveal the formation of slug flow.

\section{RESULTS AND DiSCUSSION}

\section{A. Description of the Study Cases}

In the present study, the air diffuser has two gas inlets from both sides of the pipe, and the nozzles are distributed evenly on top of the diffuser pipe. The aeration pipe is placed underneath water at a depth of $277 \mathrm{~mm}$. The water is assumed to be fouled, corresponding to a viscosity of $0.005 \mathrm{~kg} / \mathrm{m} \cdot \mathrm{s}$. Due to the geometry symmetricity, the simulation was computed with half of the air diffuser pipe with a symmetrical boundary condition in the middle. The left-hand side is the gas inlet boundary conditions, and the right-hand side is symmetry boundary. Four geometrical parameters have been studied numerically to identify the onset of slugging. The parameters are diffuser pipe diameter, nozzle diameter, diffuser pipe length and number of nozzles, the dimensions are shown in Table I.

TABLE I

PARAMETERS STUDY IN PRESENT WORK

\begin{tabular}{ccc}
\hline \hline Parameters & Units & Dimensions \\
\hline Diffuser Pipe Diameter $D_{\text {pipe }}$ & $\mathrm{mm}$ & $17.12,22.45,27.86,36.62$ \\
Nozzle Diameter $D_{\text {noz }}$ & $\mathrm{mm}$ & $3,4,5,6,7$ \\
Diffuser Pipe Length $L_{\text {pipe }}$ & $\mathrm{mm}$ & $400,500,600$ \\
Number of Nozzle $Z_{\text {noz }}$ & - & $3,4,5,6,7$ \\
\hline \hline
\end{tabular}

\section{B. Critical Gas Velocity and Flow Rate}

The critical gas velocity $U_{c}$ is defined as the minimum gas velocity where slugging will not occur, and the corresponding flow rate is called critical gas flow rate $Q_{c}$. For each simulation case, the gas inlet velocity will be decreased from a relatively large value in order to find the critical gas velocity $U_{c}$ for slugging phenomenon. The influence of the geometrical parameters on the critical gas velocity, including pipe diameter $D_{\text {pipe }}$, nozzle diameter $D_{n o z}$, pipe length $L_{p i p e}$ and number of nozzles $Z_{n o z}$ were studied and detailed below.

The effect of the pipe diameter is shown in Fig. 3. All the cases in this section have five nozzles with a pipe length of 500 $\mathrm{mm}$. As shown, the larger the pipe diameter, the lower the critical gas velocity. This indicates that the smaller pipe is easier to generate slugging flow in the aspect of the air velocity. The effect of the nozzle diameter is shown in Fig. 4. It is found that the larger the nozzle diameter, the larger the critical gas velocity. This indicates that a larger nozzle has a higher tendency to generate slug flow. It is therefore obvious that the pipe diameter plays an important role in the aspect of the critical gas velocity. The effect of the pipe length is shown in Fig. 5. The pipe length has negligible effect on the critical gas velocity. The effect of the nozzle diameter is shown in Fig. 6. The more the nozzles, the larger is the slugging velocity, and the easier slugging will happen.

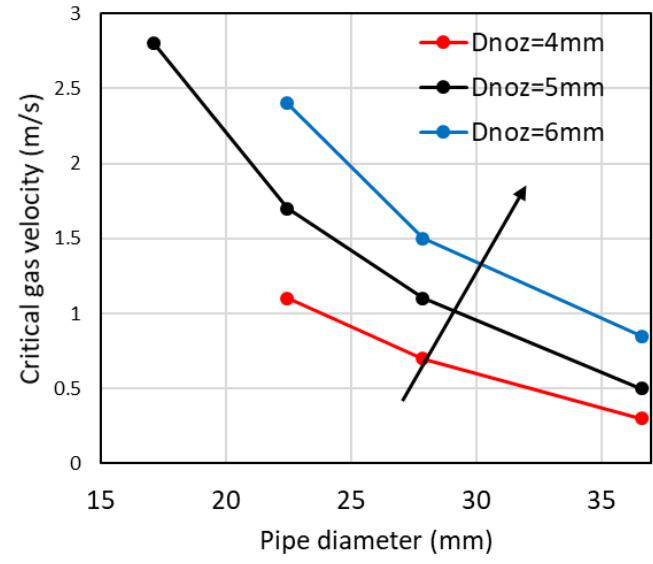

Fig. 3 Effect of the pipe diameter on the critical gas velocity $U_{c}$

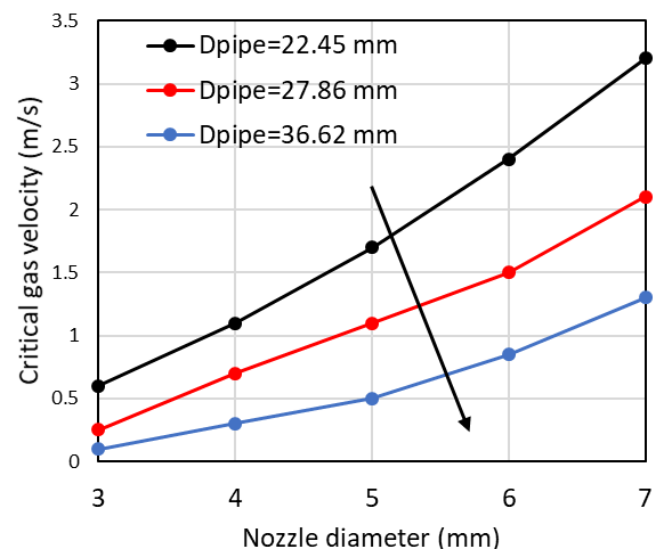

Fig. 4 Effect of the nozzle diameter on the critical gas velocity $U_{c}$

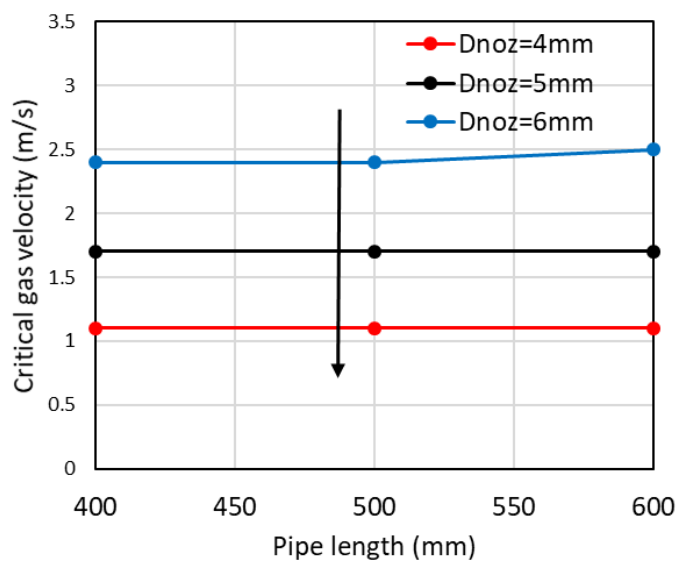

Fig. 5 Effect of the pipe length on the critical gas velocity $U_{c}$

\section{Critical Liquid Height}

The aeration pipe is fully submerged under the water with water filled initially. The liquid height will be reduced by supplying gas from the inlet. The higher gas flow rate supplies into the aeration pipe would cause the liquid height to reduce until it dropped below the critical liquid height. The critical liquid height is defined as the height of liquid when slugging happens. When the liquid height is lower than the critical liquid height, stratified flow is formed, so the air detaches through all nozzles continuously. The liquid height has been measured corresponded to its critical gas velocities in the simulation. The 
averaged liquid height underneath the first and last nozzles is reported as the liquid height in this paper. It will be affected by the geometrical parameters (e.g. $D_{p i p e}, D_{n o z}, L_{p i p e}$ and $Z_{n o z}$ ). Enlargement in aeration pipe size leads to a higher liquid height, whereas the liquid height decreases by increasing the nozzle diameter. Similarly, a reduction in liquid height is observed by increasing the number of nozzles. Last but not least, liquid height has insignificant changes with respect to the pipe length. In short, the liquid height in aeration pipe is inversely proportional to the critical gas velocity.

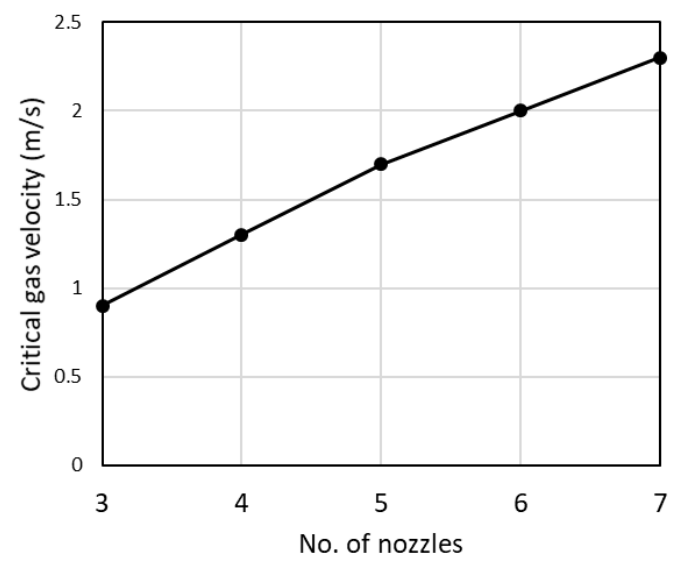

Fig. 6 Effect of the No. of nozzle on the critical gas velocity $U_{c}$

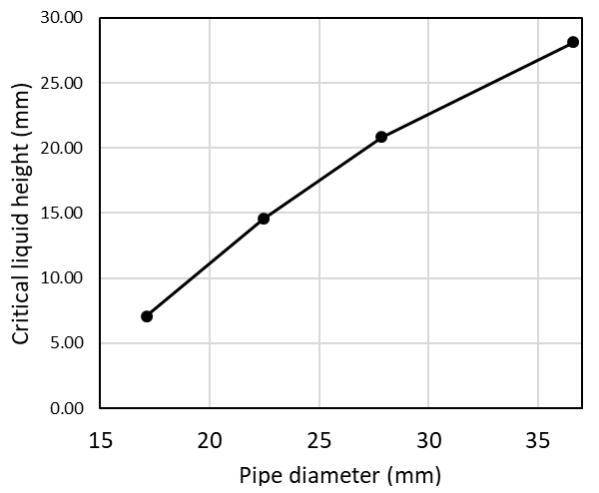

Fig. 7 Effect of the pipe diameter on the critical liquid height

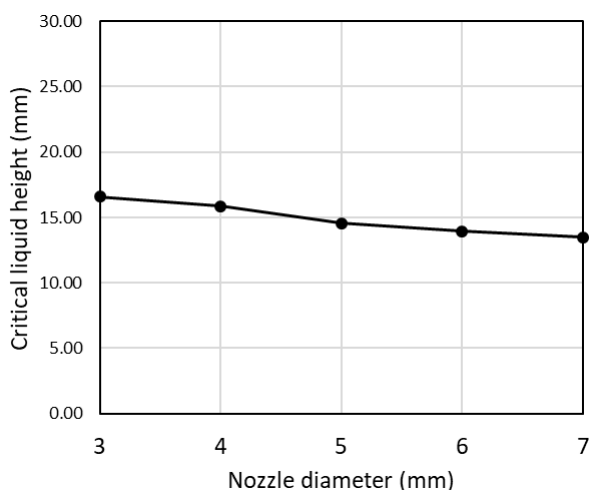

Fig. 8 Effect of the nozzle diameter on the critical liquid height

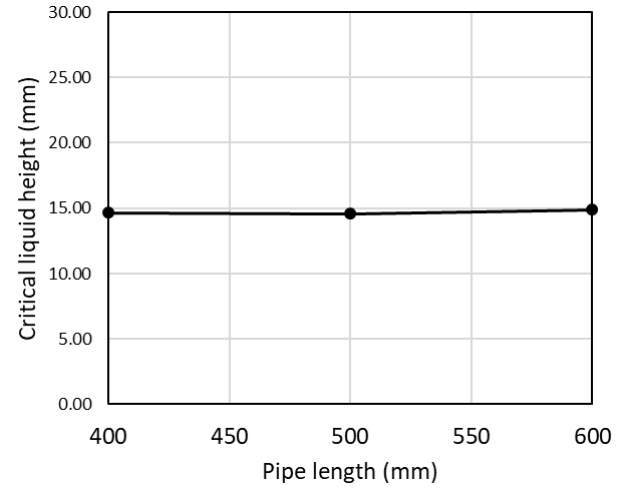

Fig. 9 Effect of the pipe length on the critical liquid height

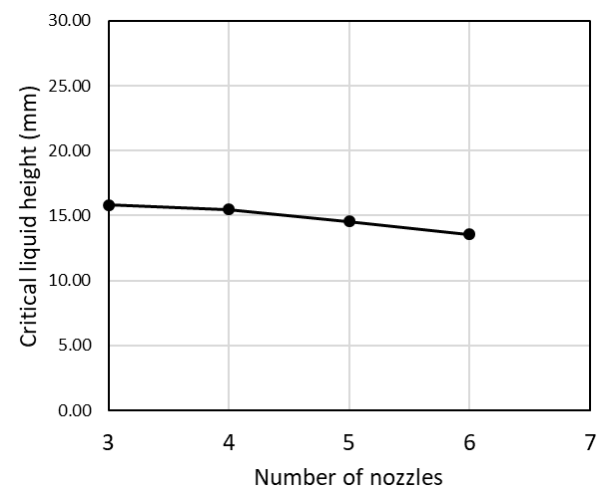

Fig. 10 Effect of the No. of nozzles on the critical liquid height

\section{D.Dimensional Analysis}

In order to conduct dimensionless analysis between critical gas velocity and geometrical parameters, a dimensionless volumetric gas flux $j_{G}^{*}$ and dimensionless void fraction $\varepsilon$ proposed by Wallis and Dobson [5] will be used. The volumetric gas flux describes the ratio of gravitational and inertia forces in the gas phase.

$$
j_{G}^{*}=\frac{\left(h_{g} / D_{\text {pipe }}\right) U_{g}}{\sqrt{\frac{\rho_{L}-\rho_{G}}{\rho_{G}} g D_{\text {pipe }}}}
$$

where $U_{g}$ is the gas velocity above the water, and $h_{g}$ is the gas height. In our simulation condition, the upper part of the righthand side equals the gas inlet velocity.

$$
\left(h_{g} / D_{\text {pipe }}\right) U_{g}=U_{g \text { inlet }}
$$

Equation 5 will become:

$$
j_{G}^{*}=\frac{U_{g \text { inlet }}}{\sqrt{\frac{\rho_{L}-\rho_{G}}{\rho_{G}} g D_{\text {pipe }}}}
$$

In the critical condition to prevent the occurrences of slug flow, where the inertia force is minimal to overcome the gravitational force (inlet gas velocity is $U_{c}$ ), the critical volumetric gas flux $j_{C, G}^{*}$ is defined as:

$$
j_{C, G}^{*}=\frac{U_{c}}{\sqrt{\frac{\rho_{L^{-}} \rho_{G}}{\rho_{G}} g D_{\text {pipe }}}}
$$


Different geometrical parameters, including pipe diameter $\mathrm{D}_{\text {pipe }}$, nozzle diameter $\mathrm{D}_{\text {noz }}$, number of nozzles $Z_{\text {noz }}$ and pipe length $L_{\text {pipe }}$ were studied in the previous sections. Results show critical gas velocity is only affected by $D_{\text {pipe }}, D_{n o z}$ and $Z_{n o z}$, which can be combined into a dimensionless parameter $A_{\text {noz }} / A_{\text {pipe. }}$. So the critical gas flux can be expressed as:

$$
j_{C, G}^{*} \propto a\left(\frac{A_{\text {noz }}}{A_{\text {pipe }}}\right)^{b}
$$

where:

$$
\begin{aligned}
& A_{\text {pipe }}=\frac{\pi}{4}\left(D_{\text {pipe }}\right)^{2} \\
& A_{\text {noz }}=\frac{\pi}{4}\left(D_{\text {noz }}\right)^{2} \times Z_{n o z}
\end{aligned}
$$

According to the simulation results, $a$ is found to be 0.5 and $b$ is 0 and $j_{C, G}^{*}=0.5 \frac{A_{\text {noz }}}{A_{\text {pipe }}}$, as shown in Fig. 11 .

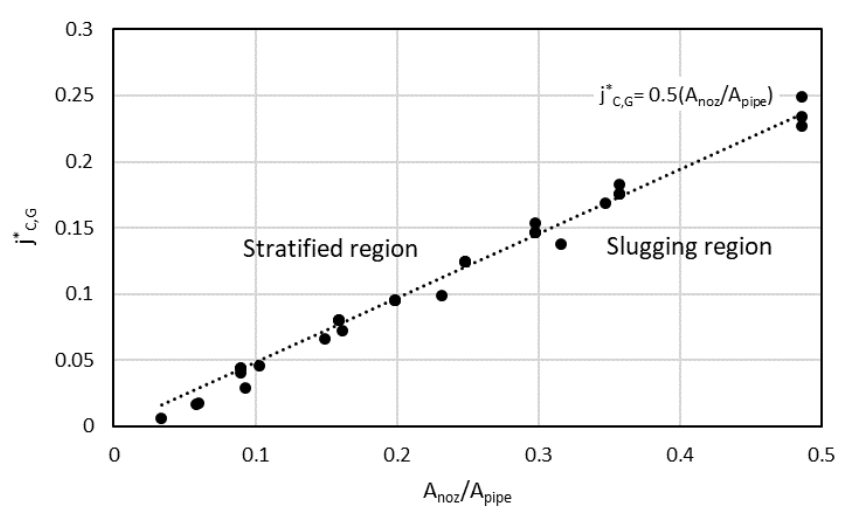

Fig. 11 The empirical model of the critical gas velocity with respect to the area ratio

The void fraction $\varepsilon$ is defined based on the height of gas phase divided by the pipe diameter, as in (13). In the critical condition of the slug flow, the critical void fraction $\varepsilon_{\mathrm{c}}$ is defined as (14).

$$
\begin{gathered}
\varepsilon=\frac{H_{G}}{D_{\text {pipe }}} \\
\varepsilon_{C}=\frac{H_{C, G}}{D_{\text {pipe }}}
\end{gathered}
$$

Literatures [4],[5] show that $j_{G}^{*}$ is linear to $\varepsilon^{1.5}$. For the critical condition of our study, we assume:

$$
j_{C, G}^{*}=A \varepsilon_{C}^{1.5}+B
$$

where $A$ and $B$ are empirical coefficients which can be obtained when the minimum RMS between results derived from (15) and simulated results happened. The pipes that we simulated has a symmetry end, and a slot outlet at the bottom of the pipe end, which is different from literature, hence the $A$ and $B$ coefficient might be different. $A$ is 1.2 and $B$ is -0.103 . The relationship between dimensionless volumetric critical gas flux and critical void fraction is shown in Fig. 12.

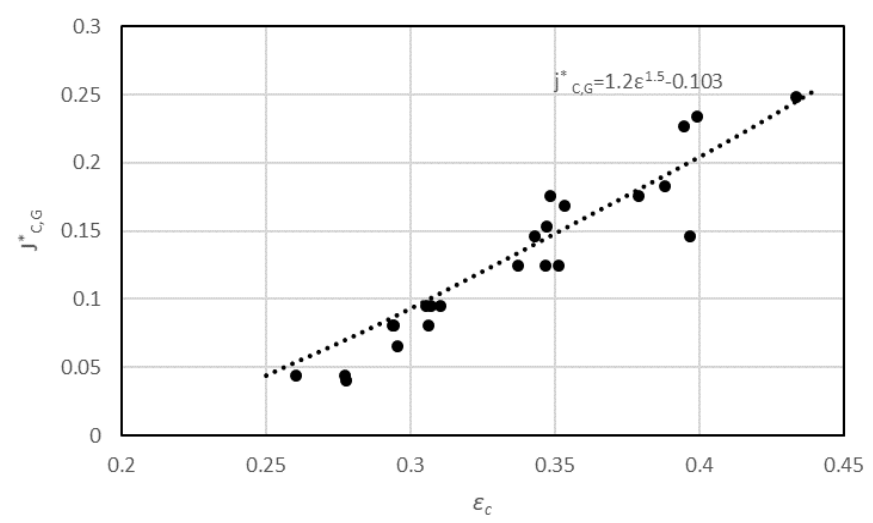

Fig. 12 The empirical model of $j^{*}{ }_{C, G}$ with respect to the $\varepsilon_{C}{ }^{1.5}$

\section{CONCLUSIONS}

Numerical simulation has been done and validated in this work. The onset of air-water slug flow in the aeration diffuser pipe in a typical membrane bioreactor has been predicted using the VOF method. The two-phase flow simulation is capable of determining the onset and subsequent development of slug flow. The effects of geometrical parameters on aeration pipe have been investigated to predict its respective critical gas velocity, which is to determine the minimum required gas flow on preventing the onset of slug flow. The critical gas velocity is found to have a strong correlation with $D_{\text {pipe }}, D_{n o z}$ and $Z_{n o z}$. The critical gas velocity, which is independent of the pipe length, increases with the increase of nozzle diameter, number of nozzles, while it decreases with increase in pipe diameter. The dimensionless critical gas flux, $j_{C, G}$, has a linear proportional relationship to the area ratio of nozzle outlet and aeration pipe inlet, with a coefficient of 0.5 . Besides, the empirical equation derived from current results between the dimensionless critical gas flux and void fraction is $j_{C, G}^{*}=1.2 \varepsilon_{C}^{1.5}-0.103$. The empirical equation would benefit the design of future aeration system to prevent the intermittency of air bubbles caused by slugging. It would improve air scouring performance on filtration membrane, thus longer lifespan on MBR system.

\section{REFERENCES}

[1] R. Sato, T. Miyayoshi, K. Hayashi and A. Tomiyama, "Effects of azimuthal angle of aeration hole on flows inside and outside an air diffuser pipe," Exp. Therm. Fluid Sci., vol. 89, pp. 90-97, Dec. 2017

[2] B.D. Woods and T.J. Hanratty, "Influence of Froude number on physical processes determining frequency of slugging in horizontal gas-liquid flows," Int. J. Multiph. Flow, vol. 25 no. 6-7, pp. 1195-1223, Sep.Nov.1999

[3] O. Dinaryanto, Y.A.K. Prayitno, A.I. Majid, A.Z. Hudaya, Y.A. Nusirwan, A. Widyaparaga, Indarto and Deendarlianto, "Experimental investigation on the initiation and flow development of gas-liquid slug two-phase flow in a horizontal pipe," Exp. Therm. Fluid Sci., vol. 81, pp. 93-108, Feb. 2017

[4] K. Mishima and M. Ishii, "Theoretical prediction of onset of horizontal slug flow," J. Fluid. Eng., vol. 102, no. 4, pp. 441-445, Dec.1980

[5] G.B. Wallis and J.E. Dodson, "The onset of slugging in horizontal stratified air-water flow," Int. J. Multiph. Flow, vol. 1, pp. 173-193, 1973 